\title{
Godot: A real-time data acquisition and control program for the PET microcomputer
}

\author{
PAUL CZERNY \\ University of Georgia, Athens, Georgia 30602
}

\begin{abstract}
This article discusses a program for the PET microcomputer that is used to record the amount of time a human infant spends attending to one of two stimuli presented via $35-\mathrm{mm}$ slides and a slide projector. The program records the number of attention episodes for either stimulus, the duration of slide presentation, and the number of slides presented. Also controlled are the transfer of data onto cassette tape at the end of the session, the detection of buttonpresses from an external keyboard, and the changing of stimuli. The interfacing used in the system and specialized subroutines are also described.
\end{abstract}

Durrett (1978) recently reviewed the Commodore PET 2001 and the Radio Shack TRS-80 personal microcomputers as laboratory instruments; two recent articles (Crossman, Williams, \& Chambers, 1978; McLean, 1978) described the use of the PET in the control of real-time experiments. The present article discusses the use of the PET to control a simple perceptual task for a human infant in real-time.

The PET, an $8 \mathrm{~K}$ version, was chosen over the TRS- 80 because of its price, real-time clock, extended version of BASIC, and interfacing capabilities.

The documentation supplied with the PET is rather limited, and the majority of the information used to implement the present system was obtained from data sheets supplied with a SYM-1 purchased from the Synertek Systems Corporation. Commodore now offers a more extensive manual than was originally supplied, but it still does not describe the logical configuration of the versatile interface adapter (VIA) integrated circuit chip (Part 6522). This chip will be discussed in the hardware section of this paper.

\section{GENERAL PROGRAM DESCRIPTION}

A flow chart of the program is given in Figure 1. The main function performed by the computer is to keep track of the time a subject spends attending to one of two stimuli presented simultaneously on $35-\mathrm{mm}$ slides and a projector. The time spent attending to a particular stimulus is defined as an attention episode; there is room to store a maximum of 30 episodes for each of 11 sets of stimuli. The start of an episode is indicated by the experimenter's pressing one of two "attention" buttons. Time is then accumulated until the same button is pressed again. The duration of each episode is stored following the second "attention" buttonpress.

The PET also controls the changing of stimuli by

Reprint requests should go to Lelon J. Peacock, Department of Psychology, University of Georgia, Athens, Georgia 30602.

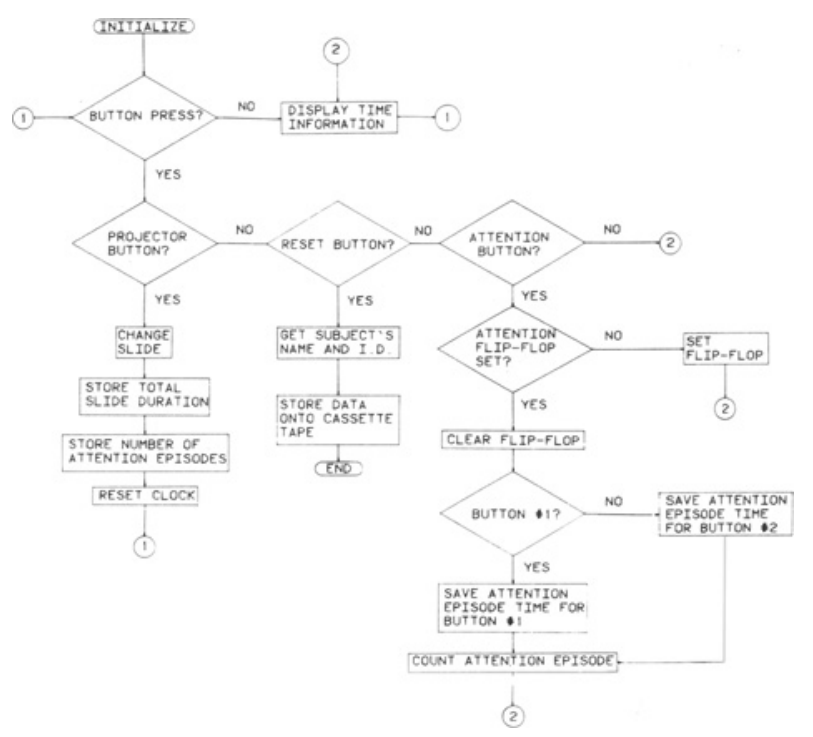

Figure 1. Program flow chart.

activating a slide projector, and it displays the duration of slide presentation and the cumulative time in seconds that the subject spends attending to a particular set of stimuli for each successive 30 -sec period. The duration of slide presentation is the time elapsed between successive "projector" buttonpresses by the experimenter. When the projector button is pressed, the duration of the slide is stored and the projector is operated via a relay. At the end of the experimental session, the data are transferred onto cassette tape.

A design error of the PET becomes obvious when large amounts of data are transferred to cassette tape: An insufficient amount of leader-trailer is placed between successive blocks of data. A block of data in the PET is 192 characters, the size of the cassette buffer. Because most data transfers require that the buffer be filled more than once, the user must compensate for this error. Documentation mentioning this 
problem was sent with some, but not all, PETs. It is discussed in the new "PET User Manual" (Commodore Business Machines, 1978), in which a solution is offered to keep count of the number of characters written to cassette. Different solutions are discussed in several PET newsletters (Laudereau, 1978; Total Information Service, 1978). The solution given here is simpler than most and is reliable on our machine.

The program in Figure 2 checks that the write to cassette takes less than $2 \mathrm{sec}$. If verified, the program assumes that the cassette buffer is still being filled and the subroutine is left. If the write takes $2 \mathrm{sec}$ or longer, it is assumed that the buffer has been emptied; more leader-trailer is put on the tape by turning on the cassette motor for six "jiffies." The motor is then turned off, and the user returns to the main program.

\section{INPUT/OUTPUT HARDWARE}

Figure 3 is a schematic drawing of the input/output (I/O) hardware. The five pushbuttons are connected to

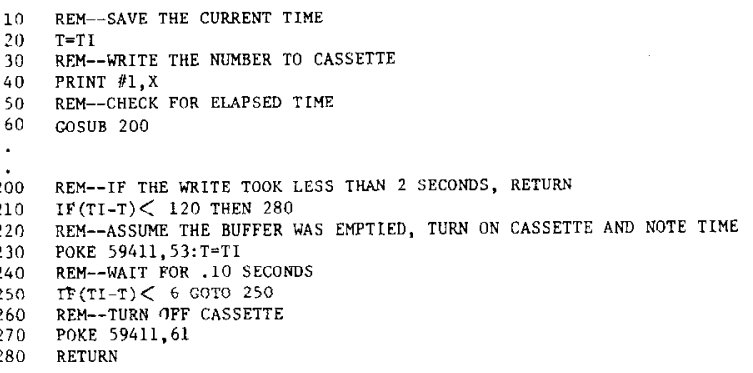

Figure 2. Subroutine to compensate for cassette buffer.

PET PARALLEL PORT EDGE CONNECTOR
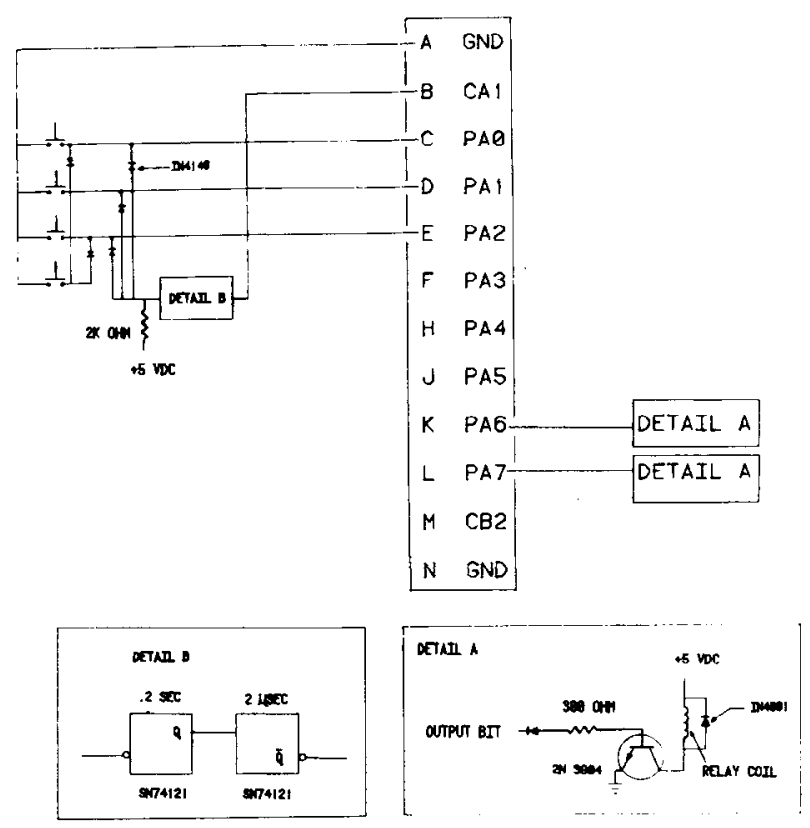

Figure 3. System input/output hardware.

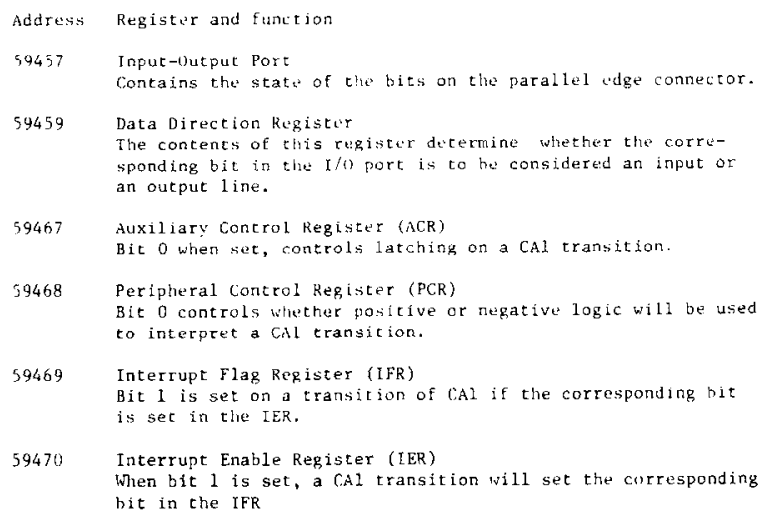

Figure 4. PET control register addresses and functions.

the I/O port of the PET and to the debouncing circuit for CA1 through a simple diode matrix. Two SN 74121 one-shot integrated circuit chips are used to debounce the line to the CAl pin. Negative logic is on the input side of the circuit; that is, a low input is true and a high input is false. Lines PA6 and PA7 are set as output lines and serve to operate two relays via transistor switches. A relay, rather than a solid state device, activates the slide projector that changes the set of stimuli, so that the same hardware configuration can be used in a number of experimental situations. For the same reason, the duration of relay operation is controlled by the program. One relay is used in this study; a second relay is included for future research.

The system configuration relies on the use of the latching capabilities of the 6522 VIA. After the appropriate instructions are given, the VIA latches the state of the bits of the I/O port when line CA1 changes state. The user has the option of specifying whether negative or positive logic is used to interpret the CAl transition, that is, for a high to low or low to high state change of CA1 to result in latching. The information is held in the $\mathrm{I} / \mathrm{O}$ port until a command is given to clear a set bit in the interrupt flag register (IFR). Figure 4 shows a list of the addresses in base 10 of the various registers used to set the VIA for latching. The function performed by each register is also given.

For the VIA to latch data, Bit 0 of the auxiliary control register (ACR) and Bit 1 of the interrupt enable register (IER) must both be set. This is accomplished by "POKEing" a 1 into the ACR and a 2 into the IER. After these bits are set, a CA1 transition causes Bit 1 of the IFR to be set and the state of the pins at the parallel connector to be latched into the $I / O$ port address. The data remain latched until Bit 1 of the IFR is cleared by POKEing a 2 into that register.

If a positive logic interpretation of the $\mathrm{CAl}$ transition is desired, Bit 0 of the peripheral control register (PCR) must be set. Again, this is accomplished by POKEing a 1 into this register. Bit 0 of the PCR is cleared when the PET is powered up. Consequently, negative logic is the default interpretation of the $\mathrm{CAl}$ transition. Advantages to using the latching capabilities of the 


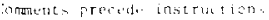

$=$ aracter to clear were

(1) 11,8

HIM $A(11,32), B(11,3, j), 14$,

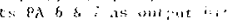

WXKE. 59459,14

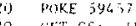

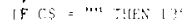

Hear interrunt: lag replitur

POKE jyohy,

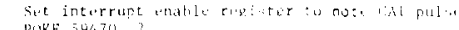

[1)KE $594+\%$,

WALT 99469,2 ?

Giear rogititer

Weliver alide and ant inite filt tialization

anstrs 590

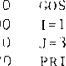

$3=\{$
PEINT

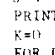

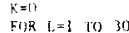

$(C, C)=1$

NEXT 1

(I) $=1$

C1ear clock

$T 1 s=" 0000000$

$x=$ PEEK $(59469)$ A.NT

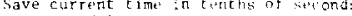

Ti=INT (T1/6)

If no pulse on Cal, displuy the tire int umat ion

if $X=0$ THEN 2000

If pulse on CAl, read l/o port and sonvert to :asitem logic

Cleas interrupt flag registe

Determine which but ton catused the flak th be sut.

and wherher this is start or and if episode.

IF $X=4$ THEN 400

$3+0$ if $X=1$ THEN 400

If reset hut ton, store litta wat: axisctle

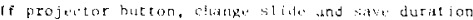

hit shide prestent
If $X=$ ? THEN 520

If any other sutlon, disilaw i imt

lioto 2000 ?

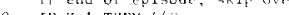

If start of attention epinde, w.t.

1) $k=1$

Then displaz time information

If end of episode, elear $h$ and wave

attention episode duratian for

respective button

$\begin{array}{ll}\text { 4दा } & K=0 \\ 650 & T 3=0\end{array}$

If but $r$ on $" 12$, ship over

IF $\mathrm{X}=1$ THFN 500

Save duration for hutcon \#1

$n(\mathrm{I}, \mathrm{J})=\operatorname{INT}((\mathrm{TL}-\mathrm{T}) / 10)$

0

Cกт० 2000

Save duration for nutton

COTO 480

If end of side prosentation, save

total duration

$A(I, 2)=\operatorname{INT}(T L / 10)$

Then change slide

COSUB 590

Cogy the information for button

$B(I, 2)-A(I, 2)$

Save the number of atcent ion episndes for

this slide

s, $A(1,1)=J-$

s60 $\quad B(1,1)=A(1,1)$

initialize episode counter and clock thelp

initialize episode counter and elock then
wat for the start of anotiter attention

episode
c0To 210

Subroutine to chinge shides

Subroutine to
Clear screet

$\$ 90$ P'RINT"CL"

uperate relay th

POKE 59457, 64: Y $\quad$ TI

WaIt for 05 secunds
IF (TI-Y) 3 Goro bio

Restore relay

$\begin{array}{ll}620 & \text { POKE } 594 \\ 630 & \text { RETIRN }\end{array}$

Program segment to get hame and slore data into cassette

Entering an asterisk will cause une characte

brark to be alliminated

然

Transferted co cisistect

700 p'ring

PRINT"ENTER TOMAY"S DATE AND SLLR.ILCT I. I1,"

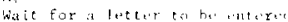

1: is: :+ $B S="$ "' ThFE

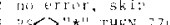

ineter lase matace

'B $1: 3,1,1$

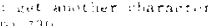

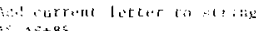

$\therefore$ sis+8s

$\because x: n: \quad s 1 x$ ing

:

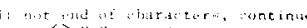

THEN 3312

- hed of haracters, dut te period

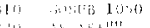

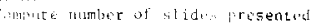

1.. to writ" data i:1to

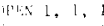

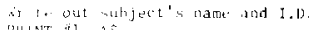

?H! WT it $\mathrm{A}$

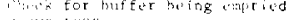

(i)

8.:.1 out nusmber of slicken presunted

$\because$ It, out ilata for huxton al

line $x=3$ or o 2

Q..... current tim

$\because 11$

ini 11 l. ght data

(1)

insilis 2086

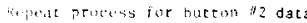

rit?

(1) ?tint \#1, $13(1, K)$

bivite 1080

VI:XT:

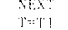

11...... out 1310

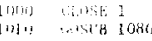

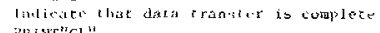

WIO PHIT"THE DATA TRANSFER IS COMPIFTE"

tivis

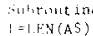

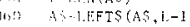

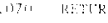

Shroutine to compensiatu for the small

Whitte huffer of the prot

It inis write to cassette took less than

losi) IF(Ti-T)< 120 THEN 1120

It 1:a butiter was umptiod, turn on the cassect

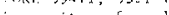

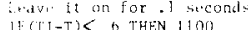

I

iurth the liassette of

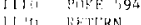
Proprag sigment to display total silde duration (wi) itinuli. The accumulated time is displaved in $\therefore$ ins that are 30 seconds wide.

cumpute the minutess and seconds that the side las thesh displayed.

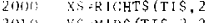

20111 Ys Miss(TIS, 3,2

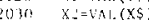

(1) isplay minutes and seconds

204(1) PRINT"FM TIME", $x_{1}, X_{2}$

cimplute amount of time sinte last displav

2051

30718

202801

$\mathrm{T}:=1 \mathrm{NT}(\mathrm{T} 1 / 10)$

bet we the in second bin

I! tleu subjuct

IF $K=0$ THEN 2190

1 efir subjece is atresding, complete accumulated

attention time for this :in

2094

2104
21101

1
$=1$

$1201+12=12-13$

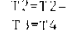

If that bin hasn't thanged since che last display, skip

Sero this time in last thin used

?hen ship

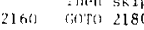

$2170 \quad C(11)=C(D)+T$

21 $211 \quad$ Dis $1=[1$

it li<20 T!IF. 2210

Qt herwise", reset peinter

$22191 \quad \mathrm{i}-20$

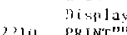

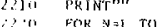

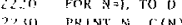

$2241 ;$ VEXT \&

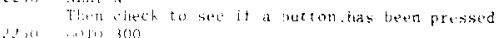

Figure 5. Godot program listing. 
VIA are that only one line, CA1, needs to be debounced and that no additional external logic is required to hold the data till they are processed; and, because Bit 1 of the IFR remains set until it is cleared, a program can alternate between processing data and checking for an event in the world outside of the CPU. Naturally, the more processing that takes place between checking for a set bit in the IFR, the longer the program recognizes that CAl was active. This is not a critical factor in our application.

\section{PROGRAM}

Figure 5 is a listing of the program. One variable, $K$, is used as a flip-flop to determine whether an attention buttonpress represents the subject's beginning or ceasing to attend to a stimulus. If the subject has started to attend, the flip-flop is set, and the program continues to display the time information. If the subject has stopped attending, the duration of the attention episode is stored, the flip-flop is cleared, and the program continues to display time information. Because only one of two possible variables for each attention episode is used, the experimenter can easily determine from the data whether or not the subject is switching his attention from one stimulus to another. After the final set of stimuli is displayed, the experimenter presses the reset button, which causes the program to store data onto cassette tape. The recording scheme used by the PET is quite slow. In fact, so much time is spent waiting for all of the data to be transferred that this program is named "Godot."

\section{BINARY CODING OF DATA}

The limit of 30 attention episodes for each of 11 sets of stimuli can be expanded by using the PEEK and POKE commands to store and retrieve data in binary coded format. This is possible because the data, as in most psychological experiments, are integers. The version of BASIC supplied with the PET requires 7 bytes of memory to store a number in floating-point format. Using 2 bytes in a binary scheme, however, allows the numbers from 0 to 65,535 to be coded. This range of numbers is probably sufficient for most psychological experiments. To convert an integer to binary form, a scheme such as that given in Figure 6 can be used.

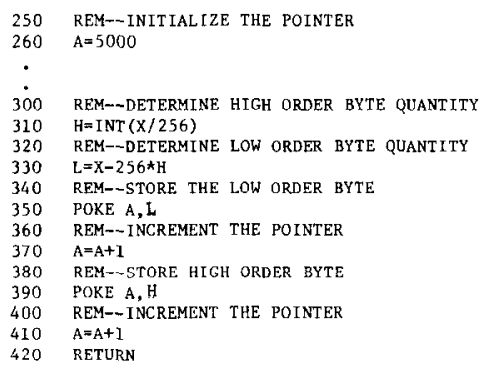

Figure 6. Floating-point to binary subroutine.

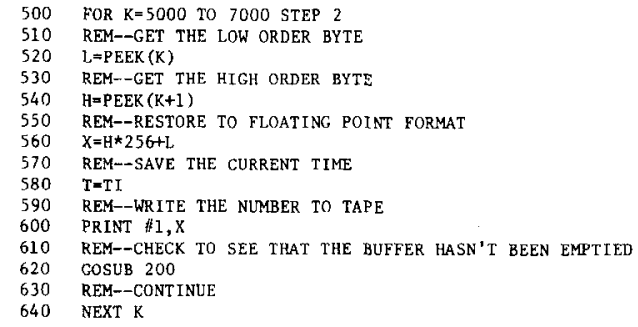

Figure 7. Binary to floating-point subroutine.

Because 1 byte in the PET can only code for the numbers from 0 to 255 , the original number $(X)$ is first divided by 256 to determine the high-order byte (HOB) quantity. Then, the HOB times 256 is subtracted from the original number. This is the low-order byte (LOB) quantity. For example, the number 526 is coded as $\mathrm{HOB}=2$ and $\mathrm{LOB}=14$. The $\mathrm{LOB}$ and $\mathrm{HOB}$ quantities can then be POKEd into two successive locations using the variable $A$ as a pointer. After all of the data are stored, they can be converted back to floating-point notation before they are written onto tape. Figure 7 is an example of the scheme. This program segment assumes that 1,000 numbers are stored starting at location 5,000 .

In the program, $\mathrm{L}$ is first set equal to the $\mathrm{LOB}$ and $\mathrm{H}$ is set equal to the HOB. $\mathrm{X}$ is then set equal to the original number and is written out to the cassette recorder. Subroutine 200 is the subroutine listed in Figure 2. PET stores the program to be executed starting at the lower part of memory and the variables used by the program starting at the higher part of memory moving toward the lower. If the use of variables is kept to a minimum, room sufficient to store 1,000 data points is then a possibility, even with a fair-sized program in core. A similar scheme can be used to read data back into core for further analysis. The numbers can then be popped (or more accurately PEEKed) out of memory one at a time and converted to floating-point notation before they are manipulated statistically.

\section{REFERENCES}

Commodore Business Machines. PET user manual. Palo Alto, Calif: Author, 1978.

Crossman, E. K., Williams, J. G., \& Chambers, J. H. Using the PET microcomputer for collecting and analyzing observational data in the classroom. Behavior Research Methods \& Instrumentation, 1978, 10, 563-566.

DuRRETT, H. J., JR. Inexpensive microcomputer systems for research and instruction: A dream or reality? Behavior Research Methods \& Instrumentation, 1978, 10, 345-351.

Laudereau, T. L. (Ed.). The paper. Audobon, Pa: ARESCO, 1978.

McLean, R. S. Using personal computers for experimental control. Behavior Research Methods \& Instrumentation, 1978, 10, 468-473.

Total Information Services. TIS workbook-PET cassette. Los Alamos, N. Mex: Author, 1978.

(Received for publication June 27, 1979; revision accepted August 15, 1979.) 